\title{
Secreted subtilisins of Microsporum canis are involved in adherence of arthroconidia to feline corneocytes
}

\begin{abstract}
Correspondence
Bernard Mignon

bmignon@ulg.ac.be
\end{abstract}

Received 18 December 2007

Accepted 7 May 2008

\author{
Aline Baldo, ${ }^{1}$ Jérémy Tabart, ${ }^{1}$ Sandy Vermout, ${ }^{2}$ Anne Mathy, ${ }^{1}$ \\ Alfred Collard, ${ }^{3}$ Bertrand Losson ${ }^{1}$ and Bernard Mignon ${ }^{1}$ \\ 1Department of Parasitology and Parasitic Diseases, Faculty of Veterinary Medicine, University of \\ Liège, B43 Sart Tilman, 4000 Liège, Belgium \\ ${ }^{2}$ Federal Agency for Medicinal and Health Products, Victor Horta Plein 40/40, 1060 Brussels, \\ Belgium \\ ${ }^{3}$ Centre d'Economie Rurale (CER), Animal Immunology, Rue du Carmel 1, 6900 Marloie, Belgium
}

\section{INTRODUCTION}

Pathogenic dermatophytes are fungi that have the ability to invade keratinized structures, such as the superficial cornified skin layers, hairs and nails, causing a superficial cutaneous infection called dermatophytosis. Microsporum canis is the main agent of dermatophytosis in cats, its natural host (Scott et al., 1995), and is responsible for a frequent zoonosis that has increased in several European countries (Lunder \& Lunder, 1992; Arrese et al., 2003). To date, little is known about the physiopathology of the infection. The first pathogenic event consists of adherence of arthroconidia to the stratum corneum, followed by their germination and invasion of the keratinized epidermis (Zurita \& Hay, 1987). The mechanisms involved in adherence of $M$. canis to epidermis have never been investigated. Most studies aiming to identify M. canis virulence factors have focused on proteases, including keratinases. A family of three subtilisins (Descamps et al., 2002), a family of five metalloproteases (Brouta et al., 2002; Jousson et al., 2004) and two dipeptidyl peptidases (GenBank accession nos. DQ286524 and DQ286525) have been identified, but so far no definitive role in fungal virulence has been assigned to these enzymes, although the in vivo secretion of several of them has been demonstrated

Abbreviations: AAPF- $p \mathrm{Na}, \quad \mathrm{N}$-succinyl-Ala-Ala-Pro-Phe-p-nitroanilide; RFE, reconstructed interfollicular feline epidermis.
(Mignon et al., 1998, 2005; Descamps et al., 2002). The role of secreted proteases in virulence has, however, been demonstrated in Candida albicans. This yeast produces a family of ten secreted aspartic proteases (Saps), which are involved in adherence and invasion of skin and mucosal surfaces (Ollert et al., 1993; Monod \& Borg-von Zepelin, 2002; Naglik et al., 2003). The role of Sap1, Sap2 and Sap3 in the adherence process has been demonstrated in several experiments using a specific aspartic protease inhibitor (Ollert et al., 1993), Sap1-3 mutant strains (Watts et al., 1998) and antibodies against Sap2, which are able to block adherence of C. albicans to epithelial cells (De Bernardis et al., 2007).

In order to gain a better understanding of the molecular mechanisms involved in $M$. canis adherence to feline epidermis, we developed an adherence model of M. canis arthroconidia to feline corneocytes, using a reconstructed interfollicular feline epidermis (RFE) (Tabart et al., 2007). This model was then used to assess the role of subtilisins, including the highly keratinolytic protease Sub3, in fungal adherence.

\section{METHODS}

Arthroconidia production. M. canis strain IHEM 21239 isolated from a naturally infected cat was used for all experiments. Arthroconidia were produced as described previously (Tabart et al., 
2007). Briefly, M. canis was grown on $2 \%$ yeast extract/ $1 \%$ peptone agar (VWR) in an atmosphere containing $12 \% \mathrm{CO}_{2}$ at $30{ }^{\circ} \mathrm{C}$ for 15 days.

\section{Detection of expression of genes encoding subtilisins in $\boldsymbol{M}$.} canis arthroconidia using nested RT-PCR. Arthroconidia were ground with a pestle and mortar under liquid nitrogen, and RNA was extracted using an RNeasy plant mini kit (Qiagen). On-column DNase digestion was performed using an RNase-free DNase set (Qiagen). RNA was eluted in $60 \mu \mathrm{l}$ water, and $5 \mu \mathrm{l}$ of the obtained solution was used as a template for RT-PCR (45 cycles) using an Access RT-PCR System (Promega) and primer pairs specific for the M. canis genes SUB1, SUB2 or SUB3, and for the actin gene as a control, as described previously (Descamps et al., 2002). In order to confirm the amplification of mRNA rather than contaminating genomic DNA, a control was performed by omission of the reverse transcriptase step in a parallel reaction. The crude reaction product $(5 \mu \mathrm{l})$ was then used as a template in a standard PCR (35 cycles) using a PCR core system I kit (Promega) and internal primers specific for M. canis actin, SUB1, SUB2 or SUB3 (Descamps et al., 2002). The expected amplicon lengths for these genes were 282, 388, 1010 and 480 bp for M. canis, respectively.

\begin{abstract}
Adherence assays. Adherence assays were performed on an RFE comprising a fully differentiated epidermis built up with feline keratinocytes seeded on a dermal equivalent, as described previously (Tabart et al., 2007). Briefly, feline fetal fibroblasts and keratinocytes were cultured as monolayers in fibroblast growth medium (FGM) and keratinocyte growth medium (KGM; BioWhittaker), respectively, and used at second passage. Fibroblasts were adjusted to $1 \times 10^{3}$ cells ml $^{-1}$ in FGM containing $2 \mathrm{mg}$ rat tail collagen $\mathrm{ml}^{-1}$ (Roche Applied Science). The cellular suspension was poured onto a cell insert (Anopore, $0.63 \mathrm{~cm}$ diameter, $0.2 \mu \mathrm{m}$ diameter pore size; VWR) supplied with a stainless steel ring, and the cell inserts were immersed in FGM for $24 \mathrm{~h}$. The medium was then replaced with KGM, whilst proliferating keratinocytes were seeded onto the dermal equivalent in cell inserts at $5 \times 10^{5}$ cells $\mathrm{cm}^{-2}$. After immersion for $24 \mathrm{~h}$ in $\mathrm{KGM}$, the cell inserts were lifted to the air-liquid interface, and the medium was replaced with RFE culture medium (Tabart et al., 2007) and changed every other day for 14 days. As the RFE was lifted to the interface, exchanges between reconstructed epidermis and the culture medium occurred only through the dermal equivalent. Twenty-four hours before RFE inoculation, penicillin, streptomycin and Fungizone were removed from the RFE culture medium.
\end{abstract}

For adherence assays, a $20 \mu \mathrm{l}$ arthroconidial suspension $\left(5 \times 10^{6}\right.$ cells $\mathrm{ml}^{-1}$ in PBS) was spread over the surface of the RFE and co-cultures were incubated in a humidified atmosphere containing $5 \% \mathrm{CO}_{2}$ for 0 , 2,4 or $6 \mathrm{~h}$ at $37^{\circ} \mathrm{C}$. After washing in PBS, conidia that were adherent to RFE were labelled using Calcofluor White $(20 \mu \mathrm{g}$ in water; fluorescent brightener 28; Sigma) (Ruchel \& Schaffrinski, 1999) and counted under a fluorescence light microscope (440 nm; Nikon; Fig. $1)$. The experiment was performed in triplicate and the incubation time was unknown to the observer.

Production of mAbs. Recombinant $31.5 \mathrm{kDa}$ keratinolytic subtilisin (rSub3) was purified by cation-exchange chromatography from the supernatant of a transformed Pichia pastoris colony (Descamps et al., 2003) and dialysed against PBS. BALB/c (Sp2/0) mice were immunized subcutaneously on days $0,21,42$ and 59 using $50 \mu \mathrm{g}$ rSub3 mixed with Quil A adjuvant (Isconova). On day 61, mice were euthanized. Spleen cells $\left(3.5 \times 10^{6}\right.$ cells $\left.\mathrm{ml}^{-1}\right)$ were suspended in RPMI 1640 (Cambrex) and mixed with myeloma cells (SP2/0-Ag 14, ATCC CRL $8287,1.7 \times 10^{6}$ cells $\mathrm{ml}^{-1}$ ) in the presence of PEG (Sigma). Cells were then centrifuged for $5 \mathrm{~min}$ at $1000 \mathrm{~g}$, suspended in a selective medium (RPMI 1640 containing $10 \%$ fetal calf serum and hypoxanthine, aminopterin and thymidine) and distributed into

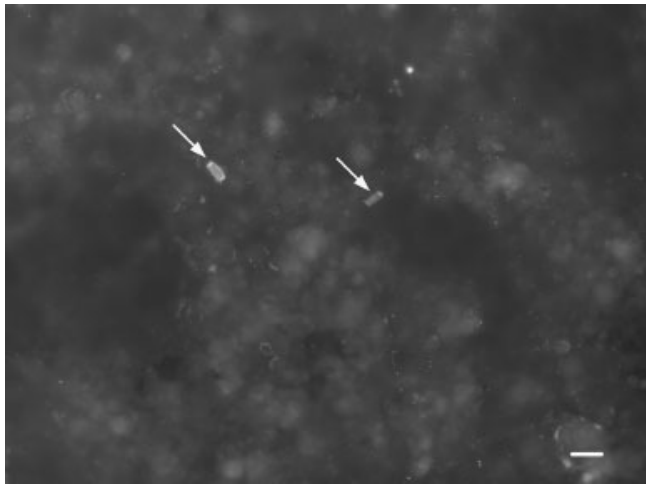

Fig. 1. Adherence model for $M$. canis IHEM 21239 on RFE. Adherent arthroconidia (indicated by arrows) were stained using Calcofluor White and visualized under a fluorescence light microscope $(440 \mathrm{~nm})$. Bar, $5 \mu \mathrm{m}$.

96-well microplates in the presence of mouse peritoneal cells as a feeder layer. Secretion of rSub3 antibodies was assessed by ELISA. After cloning by limiting dilution, mAbs were produced in flasks and purified by affinity chromatography using protein A (Sigma) and evaluated for their ability to inhibit the activity of rSub3 towards the synthetic substrate $N$-succinyl-Ala-Ala-Pro-Phe- $p$-nitroanilide (AAPF- $p$ Na; Sigma) as described previously (Mignon et al., 1998). Two mAbs were selected (6B8 and 4F10) and Western blotting was performed to demonstrate their specific binding to Sub3. In addition to rSub3, a crude exo-antigen containing Sub3 and many other secreted proteases of $M$. canis was used. This exo-antigen was prepared as described previously (Mignon et al., 1998). Briefly, $M$. canis strain IHEM 21239 was grown in a liquid medium containing cat keratin, and the culture supernatant was harvested after 15 days and referred to as crude exo-antigen. rSub3 was used as a positive control in a Western blot. Following separation of the proteins by $12 \%$ SDS-PAGE, the gel was electroblotted for $1 \mathrm{~h}$ at $45 \mathrm{~mA}$ onto PVDF membrane in Tris/glycine buffer with $20 \%$ methanol, and then treated with a mixture of the two mAbs and horseradish peroxidaseconjugated rabbit anti-mouse Ig (Dako). The blots were treated with the chemiluminescent detection reagent ECL Plus (Amersham Pharmacia) and were exposed to film.

Inhibition of adherence. The serine protease inhibitor chymostatin (Sigma), a cocktail of protease inhibitors (protease inhibitor cocktail for use in tissue culture medium with a broad specificity for serine, cysteine and acid proteases and for aminopeptidases) (Sigma) supplemented with chymostatin, and a mixture of the two antiSub3 mAbs $(1: 1)$ were tested for their capacity to inhibit the adherence of $M$. canis arthroconidia to RFE. Chymostatin was dissolved in $10 \mathrm{mM}$ DMSO and used at a final concentration of $100 \mu \mathrm{M}$ in PBS. The cocktail of protease inhibitors stored in DMSO by the manufacturer was diluted 100 -fold in PBS. The mAb mixture was used at a concentration of $500 \mu \mathrm{g} \mathrm{ml}^{-1}$ in PBS. Adherence assays were performed as described above except that arthroconidia were exposed to the inhibitors for $1 \mathrm{~h}$ prior to the adherence assay. Chymostatin, the cocktail of protease inhibitors supplemented with chymostatin or the mAbs were also added to the RFE culture medium at the same final concentration as above. A control without inhibitors or mAbs was also used under the same conditions. An additional control with a non-specific mAb (mouse mAb AA5H to influenza virus nucleoprotein; Abcam) at the same concentration was also performed. Infected RFE were incubated for $4 \mathrm{~h}$. The experiment was 
performed in triplicate and the status of each RFE was unknown to the observer.

Expression of results and statistical analysis. Inhibition of $M$. canis adherence was expressed as the residual percentage of adherence to RFE in comparison with the controls. Significant differences between two sets of data were calculated using Student's $t$-test, with significance defined as $P<0.005$.

\section{RESULTS AND DISCUSSION}

\section{Detection of SUB1 and SUB3 mRNAs in arthroconidia}

The SUB1 and SUB3 genes, but not SUB2, were found to be transcribed in arthroconidia (Fig. 2), strongly suggesting that the corresponding encoded proteases are produced in these spores. Arthroconidia were used because they constitute the infective stage of $M$. canis in vivo. They are shed from infected lesions and disseminated into the environment. Consequently, it can be postulated that the proteases Sub1 and Sub3 are involved in the early stages of infection, i.e. adherence to and/or invasion of corneocytes.

\section{M. canis arthroconidia adhere to RFE up to $6 \mathrm{~h}$ post-inoculation}

In the present model, adherence of arthroconidia to RFE was found to be time-dependent (Fig. 3), starting within $2 \mathrm{~h}$ and increasing up to $6 \mathrm{~h}$ post-inoculation (the last observation time). This is, to the best of our knowledge, the first report of a model to study $M$. canis adherence to epidermis. The kinetics of adherence was similar to that reported for other dermatophytes. Zurita \& Hay (1987)

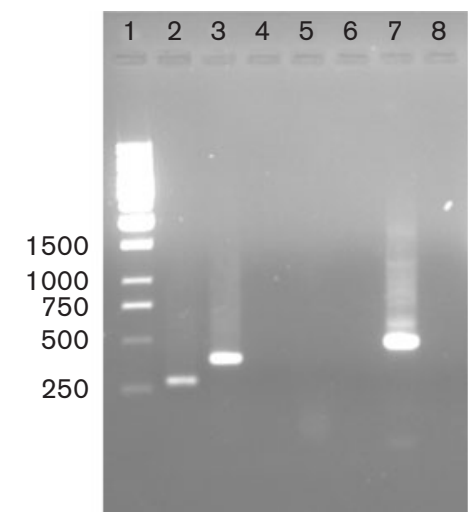

Fig. 2. Transcription of $M$. canis IHEM 21239 SUB1 and SUB3 in arthroconidia. Lane 1, molecular mass markers (1 kb ladder; Fermentas), sizes (in bp) are indicated on the left; lanes 2, 3, 5 and 7 , nested RT-PCR products obtained from arthroconidia, corresponding to internal cDNA fragments of the $M$. canis actin gene, SUB1, SUB2 and SUB3, respectively; lanes 4, 6 and 8, control reactions (with the reverse transcriptase step omitted), corresponding to $M$. canis SUB1, SUB2 and SUB3, respectively.

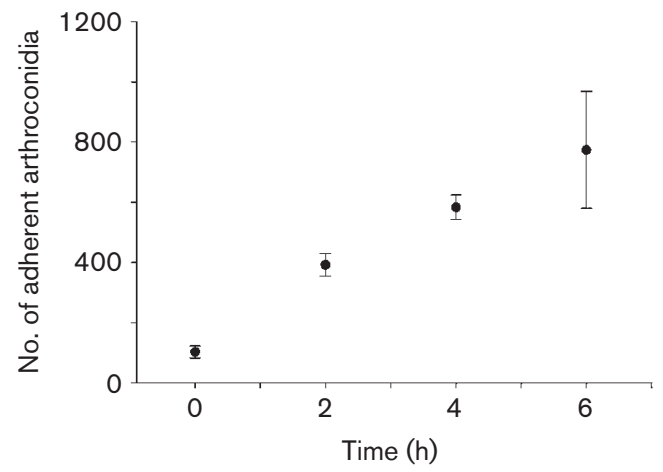

Fig. 3. Kinetics of $M$. canis IHEM 21239 adherence to RFE. Results are shown as the mean number $( \pm S D)$ of adherent arthroconidia.

observed that maximum adherence of Trichophyton sp. arthroconidia to human keratinocytes in suspension occurred within 3-4 h. Aljabre et al. (1992, 1993) used stripped sheets of stratum corneum or isolated keratinocytes to demonstrate that adherence of Trichophyton mentagrophytes arthroconidia was highest $6 \mathrm{~h}$ after contact and that germination of these spores began $4 \mathrm{~h}$ postexposure. Adherence of T. mentagrophytes to human skin explants of full epidermis was maximal after $12 \mathrm{~h}$ (Duek et al., 2004). In the present study, adherence of M. canis arthroconidia was studied using RFE. This model is likely to be very useful because both the cornified layer resulting from the epidermal differentiation process (Tabart et al., 2007) and skin permeability of RFE were shown to be comparable to the in vivo situation.

\section{M. canis adherence to RFE is inhibited by chymostatin and mAbs against Sub3}

Chymostatin, a serine protease inhibitor that completely inhibits Sub3 activity (Mignon et al., 1998), significantly inhibited the adherence of $M$. canis arthroconidia to RFE ( $53 \%$ inhibition, $P<0.005$, Fig. 4 ). In the yeast $C$. albicans, pepstatin $\mathrm{A}$, an aspartic protease inhibitor, decreased $C$. albicans adherence to keratinocytes (45\% inhibition; Ollert et al., 1993), and strongly reduced tissue damage caused to the vaginal and oral epithelia by C. albicans cells (Schaller et al., 1999, 2003). A reduced adherence of Sap1-, Sap2and Sap3-deficient strains to buccal epithelial cells has also been observed (Watts et al., 1998). The inhibition of $M$. canis adherence to RFE by chymostatin clearly indicated that one or more subtilisins play an important role in $M$. canis adherence. The cocktail of protease inhibitors supplemented with chymostatin decreased the adherence of arthroconidia to RFE by $72 \%$ (Fig. 4), suggesting that other proteases are also involved in the adherence process.

Two mAbs against Sub3 (6B8 and 4F10) were produced and purified. Western blotting showed that these mAbs were specific to Sub3 and did not cross-react with other 


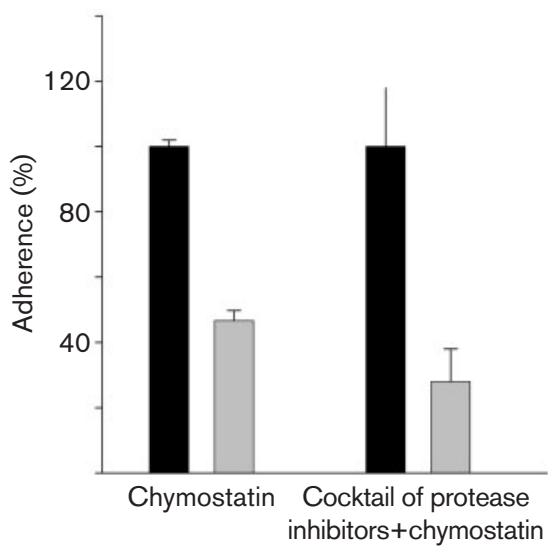

Fig. 4. Adherence of $M$. canis IHEM 21239 arthroconidia to RFE in the presence of chymostatin or chymostatin plus a cocktail of protease inhibitors. Chymostatin was used at a final concentration of $100 \mu \mathrm{M}$ and the cocktail of protease inhibitors at a 100 -fold dilution. Adherence values in the presence of inhibitors (grey bars) are expressed as a percentage of the controls without inhibitor $(100 \%)$ (black bars) and are given as means \pm SD.

secreted proteases (Fig. 5). The mAbs did not inhibit rSub3 activity towards AAPF- $p \mathrm{Na}$ (Fig. 6). However, they significantly inhibited adherence of arthroconidia to RFE by $23 \%(P<0.005$; Fig. 7$)$, indicating that inhibition could be due to steric hindrance rather than blockage of the active site. The non-specific control mAb did not inhibit adherence (Fig. 7), excluding non-specific steric effects of mAbs against Sub3. Cross-reactivity of the mAbs against Sub3 with Sub1 and Sub2 is unlikely, as Sub1 and Sub3 have only $41.2 \%$ identity, and Sub2 and Sub3 have only $34.6 \%$ identity (Descamps et al., 2002). Overall, these results suggest that, among the subtilisins, Sub3 is

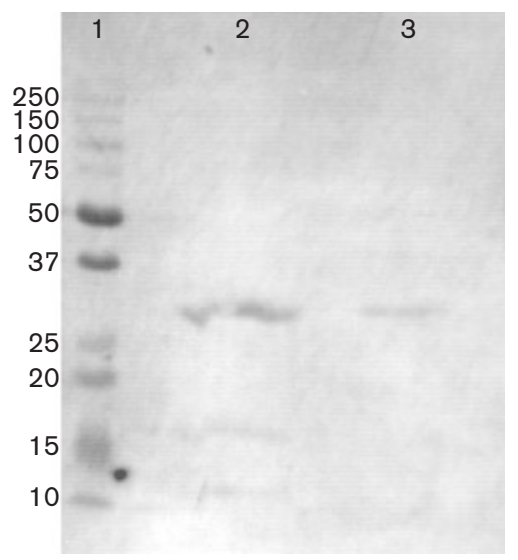

Fig. 5. Western blot showing the specificity of a mixture of mAbs 4F10 and 6B8 for Sub3. Lane 1, molecular mass markers (Precision Plus dual colour protein standards; Bio-Rad), sizes in $\mathrm{kDa}$ are indicated on the left; lane 2, rSub3; lane 3, exo-antigen.

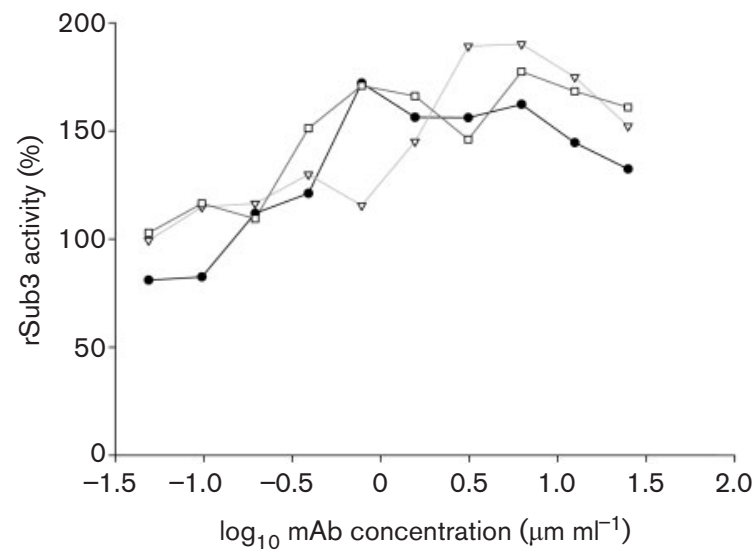

Fig. 6. Activity of rSub3 towards AAPF- $p N a$ in the presence of anti-Sub3 mAbs 6B8 and 4F10. Activity values are expressed as a percentage of the activity of $r \mathrm{Sub} 3$ towards AAPF- $p N a$ without mAbs (100\%). •, 6B8; $\nabla, 4 \mathrm{~F} 10 ; \square, 6 \mathrm{~B} 8+4 \mathrm{~F} 10$.

implicated in the adherence mechanisms. The precise mechanisms by which secreted proteases contribute to the adherence process are not clear. Two hypotheses are currently favoured (Naglik et al., 2003). Firstly, proteins could act as ligands for surface moieties on host cells, which does not necessarily require activity of these enzymes. In the pathogenic bacteria Porphyromonas gingivalis and Streptococcus pneumoniae, a membraneassociated dipeptidyl peptidase IV and a surface-associated serine protease named choline-binding protein $\mathrm{G}$, respectively, have been implicated in adherence (Kumagai et al.,

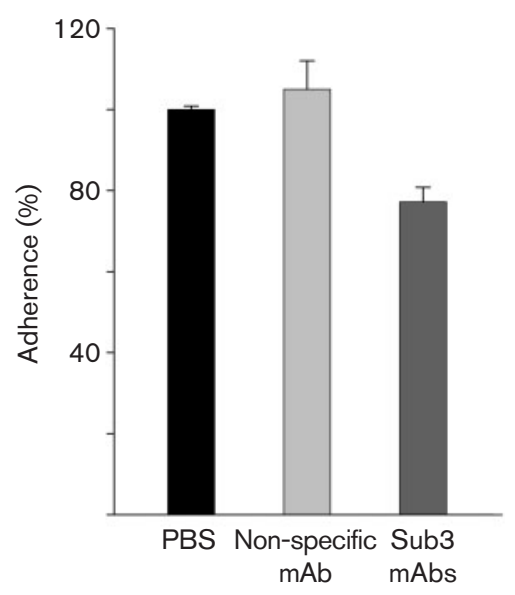

Fig. 7. Adherence of $M$. canis IHEM 21239 arthroconidia to RFE in the presence of anti-Sub3 mAbs (6B8 and 4F10). The mAbs were used at a final concentration of $500 \mu \mathrm{g} \mathrm{ml}^{-1}(25 \mu \mathrm{g}$ each $\mathrm{mAb}$ per RFE). A non-specific control $\mathrm{mAb}$ was used the same concentration. Adherence values are expressed as a percentage of the control test without mAbs (PBS only; $100 \%$ ) and are given as the mean $\pm S D$. 
2005; Mann et al., 2006). Interestingly, the carboxylterminal extremity of Sub1 shows a remarkable abundance of proline-rich domains (Descamps et al., 2002), suggesting that this protein could also be surface anchored. Secondly, secreted proteases may act as active enzymes to modify target ligands on the fungal surface or on epithelial cells, and this could lead to conformational changes, facilitating adherence of the fungus (Monod \& Borg-von Zepelin, 2002). Our study on M. canis adherence mechanisms could lead to the implementation of new therapeutic tools aimed at preventing the adherence of arthroconidia to keratinocytes.

\section{ACKNOWLEDGEMENTS}

The authors thank Drs M. Blaise, O. Delpire and M. Simon for their cooperation in this study. Many thanks also to Mrs B. Robert and to the Centre d'Economie Rurale (CER) of Marloie for their help with $\mathrm{mAb}$ production. Also they sincerely thank veterinarians of the Prince Laurent Foundation (Seraing, Belgium), Dr B. Adam and the Department of Obstetrics and Reproduction (Faculty of Veterinary Medicine, University of Liège). This work was supported by grant 3.4595.04 from Fonds de la Recherche Scientifique Médicale (FRSM). A.B., J.T. and A.M. are the recipients of studentships from FRIA (Fonds pour la Formation à la Recherche dans l'Industrie et dans l'Agriculture, 1000 Bruxelles, Belgium).

\section{REFERENCES}

Aljabre, S. H., Richardson, M. D., Scott, E. M. \& Shankland, G. S. (1992). Germination of Trichophyton mentagrophytes on human stratum corneum in vitro. J Med Vet Mycol 30, 145-152.

Aljabre, S. H., Richardson, M. D., Scott, E. M., Rashid, A. \& Shankland, G. S. (1993). Adherence of arthroconidia and germlings of anthropophilic and zoophilic varieties of Trichophyton mentagrophytes to human corneocytes as an early event in the pathogenesis of dermatophytosis. Clin Exp Dermatol 18, 231-235.

Arrese, J. E., Piérard-Franchimont, C. \& Piérard, G. E. (2003). Les teignes du cuir chevelu d'ici et d'ailleurs. Quand la prévention est à géographie variable. Rev Med Liege 58, 388-391.

Brouta, F., Descamps, F., Monod, M., Vermout, S., Losson, B. \& Mignon, B. (2002). Secreted metalloprotease gene family of Microsporum canis. Infect Immun 70, 5676-5683.

De Bernardis, F., Liu, H., O’Mahony, R., La Valle, R., Bartollino, S., Sandini, S., Grant, S., Brewis, N., Tomlinson, I. \& other authors (2007). Human domain antibodies against virulence traits of Candida albicans inhibit fungus adherence to vaginal epithelium and protect against experimental vaginal candidiasis. J Infect Dis 195, 149-157.

Descamps, F., Brouta, F., Monod, M., Zaugg, C., Baar, D., Losson, B. \& Mignon, B. (2002). Isolation of a Microsporum canis gene family encoding three subtilisin-like proteases expressed in vivo. J Invest Dermatol 119, 830-835.

Descamps, F., Brouta, F., Vermout, S., Monod, M., Losson, B. \& Mignon, B. (2003). Recombinant expression and antigenic properties of a $31.5-\mathrm{kDa}$ keratinolytic subtilisin-like serine protease from Microsporum canis. FEMS Immunol Med Microbiol 38, 29-34.

Duek, L., Kaufman, G., Ulman, Y. \& Berdicevsky, I. (2004). The pathogenesis of dermatophyte infections in human skin sections. $J$ Infect 48, 175-180.
Jousson, O., Lechenne, B., Bontems, O., Capoccia, S., Mignon, B., Barblan, J., Quandroni, M. \& Monod, M. (2004). Multiplication of an ancestral gene encoding secreted fungalysin preceded species differentiation in the dermatophytes Trichophyton and Microsporum. Microbiology 150, 301-310.

Kumagai, Y., Yagishita, H., Yajima, A., Okamoto, T. \& Konishi, K. (2005). Molecular mechanism for connective tissue destruction by dipeptidyl aminopeptidase IV produced by the periodontal pathogen Porphyromonas gingivalis. Infect Immun 73, 2655-2664.

Lunder, M. \& Lunder, M. (1992). Is Microsporum canis infection about to become a serious dermatological problem? Dermatology 184, 8789.

Mann, B., Orihuela, C., Antikainen, J., Gao, G., Sublett, J., Korhonen, T. K. \& Tuomanen, E. (2006). Multifunctional role of choline binding protein $G$ in pneumococcal pathogenesis. Infect Immun 74, 821-829.

Mignon, B., Swinnen, M., Bouchara, J. P., Hofinger, M., Nikkels, A., Pierard, G., Gerday, C. \& Losson, B. (1998). Purification and characterization of a $31.5-\mathrm{kDa}$ keratinolytic subtilisin-like serine protease from Microsporum canis and evidence of its secretion in naturally infected cats. Med Mycol 36, 395-404.

Mignon, B., Vermout, S., Brouta, F., Nikkels, A., Losson, B. \& Descamps, F. (2005). In vivo mRNA expression analysis of Microsporum canis secreted subtilisin-like serine proteases in feline dermatophytosis. In Advances in Veterinary Dermatology, vol. 5, pp. 255-260. Edited by A. Hillier, A. P. Foster \& K. W. Kwochka. Oxford: Blackwell Publishing.

Monod, M. \& Borg-von Zepelin, M. (2002). Secreted proteinases and other virulence mechanisms of Candida albicans. Chem Immunol 81, $114-128$.

Naglik, J. R., Challacombe, S. J. \& Hube, B. (2003). Candida albicans secreted aspartyl proteinases in virulence and pathogenesis. Microbiol Mol Biol Rev 67, 400-428.

Ollert, M. W., Sohnchen, R., Korting, H. C., Ollert, U., Brautigam, S. \& Brautigam, W. (1993). Mechanisms of adherence of Candida albicans to cultured human epidermal keratinocytes. Infect Immun 61, 45604568.

Ruchel, R. \& Schaffrinski, M. (1999). Versatile fluorescent staining of fungi in clinical specimens by using the optical brightener Blankophor. J Clin Microbiol 37, 2694-2696.

Schaller, M., Korting, H. C., Schafer, W., Bastert, J., Chen, W. \& Hube, B. (1999). Secreted aspartic proteinase (Sap) activity contributes to tissue damage in a model of human oral candidosis. Mol Microbiol 34, 169-180.

Schaller, M., Bein, M., Korting, H. C., Baur, S., Hamm, G., Monod, M., Beinnhauer, S. \& Hube, B. (2003). The secreted aspartyl proteinases Sap1 and Sap2 cause tissue damage in an in vitro model of vaginal candidiasis based on reconstituted human vaginal epithelium. Infect Immun 71, 3227-3234.

Scott, D. W., Miller, W. H. \& Griffin, C. E. (1995). Fungal skin diseases. In Small Animal Dermatology, 5th edn, pp. 329-391. Edited by G. H. Muller. Philadelphia, PA: W. B. Saunders.

Tabart, J., Baldo, A., Vermout, S., Nusgens, B., Lapiere, C., Losson, B. \& Mignon, B. (2007). Reconstructed interfollicular feline epidermis as a model for Microsporum canis dermatophytosis. J Med Microbiol 56, 971-975.

Watts, H. J., Cheah, F. S. H., Hube, B., Sanglard, D. \& Gow, N. A. R. (1998). Altered adherence in strains of Candida albicans harbouring null mutations in secreted aspartic proteinase genes. FEMS Microbiol Lett 159, 129-135.

Zurita, J. \& Hay, R. J. (1987). Adherence of dermatophyte microconidia and arthroconidia to human keratinocytes in vitro. $J$ Invest Dermatol 89, 529-534. 